Sharif University of Technology
Scientia Iranica
Transactions E: Industrial Engineering
wCIENTIA

Research Note

\title{
Intelligent choice-based network revenue management
}

\author{
F. Etebari $\mathbf{i}^{\mathrm{a}, *}$ and A.A. Najafi ${ }^{\mathrm{b}}$ \\ a. Faculty of Industrial and Mechanical Engineering, Qazvin Branch, Islamic Azad University, Qazvin, Iran. \\ b. Department of Industrial Engineering, K.N. Toosi University of Technology, Tehran, Iran. \\ Received 22 November 2013; received in revised form 10 January 2015; accepted 4 May 2015
}

\section{KEYWORDS}

Choice-based network revenue management; Choice model; Optimization module; Interaction; Knowledge acquisition; Artificial neural network.

\begin{abstract}
Choice-based network revenue management concentrates on importing choice models within the traditional revenue management system. Multinomial logit is a popular and well-known model which is the basic choice model in revenue management. Empirical results indicate inadequacy of this model for predicting itinerary shares; therefore, more realistic models, such as nested logit, can be proposed for substituting it. Incorporating complex choice models in the optimization module based on statistical tests without considering the complexity of the obtained mathematical model would lead to increase in the complexity of a system without obtaining significant improvement. Considering the influence of discrete choice model on the structure of optimization model, it is necessary to analyze the interaction between specific discrete choice and optimization models. In this paper, a knowledge acquisition subsystem is introduced for providing intelligence and considering the most suitable choice models. We develop the feedforward multilayer perceptron artificial neural network for forecasting revenue improvement percent obtained by using more realistic choice models. The obtained results demonstrate that the new system will decrease the complexity of the system, simultaneously, while preserving revenue of the firm. According to the computational results, by increasing the resource restriction, the process of incorporating more realistic choice model will be more important.

(C) 2016 Sharif University of Technology. All rights reserved.
\end{abstract}

\section{Introduction}

Revenue management is defined to predict behavior of the consumer at the micro market level and be used for optimizing availability and price of products to maximize revenue of the firm [1]. One of the most restrictive assumptions of the traditional revenue management models is independent demand assumption. Regarding this assumption, demand is considered as a sequence of requests for products which are insensitive to the capacity controls applied by the firm and market conditions [2]. In order to relax this restriction, it is proposed to apply choice-based revenue management models. The most important challenges

*. Corresponding author. Tel.: +982833665275

E-mail address: featebari@gmail.com (F. Etebari) of these models are choosing realistic discrete choice models and keeping complexity of the optimization problem at a reasonable level. Applying more complex and realistic choice model poses major computational challenges for operation researchers [3]. Balancing additional expected potential revenue obtained by incorporating more complex and realistic choice model in the optimization module with time complexity of the obtained optimization problem is a main motivation of this paper. The intelligent decision support system is proposed for this purpose.

In the literature, most of the research about the choice-based revenue management models concentrate on the development of optimization models according to the general structure of choice-based models. Belobaba and Hopperstad [4] studied purchase behavior of consumers by simulation for considering the impact 
of airline schedule, path, and fare on preferences of passengers. Zhang and Cooper [5] formulated seatinventory control of a set of parallel flights under customer choice using Markov decision processes. Virtual nesting control strategy is studied in a quantity-based network revenue management problem with substitutable products [6]. Etebari et al. [7] developed choicebased network revenue management models by more realistic discrete choice models.

The list of products preferred by customers is analyzed as preference orders [8]. An efficient sets concept for single leg revenue management model is introduced by Talluri \& van Ryzin [9]. Deterministic linear programming model is introduced for solving choice-based revenue management models [10]. Previous work was extended by allowing market segmentation to the choice model by disjoint consideration sets of products [11]. They extended the efficient sets concept to network and showed that as demand and capacity are scaled up asymptotically, only efficient sets are used in an optimal policy. Bront et al. [12] extended work of Liu \& Van Ryzin [11] by applying market segmentation in a way that products could belong to overlapping segments. The heuristics for improving initial guess of bid price vector is considered by Meissner and Strauss [13] in network revenue management.

Incorporating realistic choice model in the optimization module and solving the obtained problem are two main stages of revenue management models. Multinomial logit model is a well-known popular choice model. Independence of Irrelevant Alternatives (IIA) implies that the ratio of choice probabilistic is independent from the attributes of any other alternatives. The nested logit model incorporates more realistic substitution patterns by relaxing the independence assumption of error terms of the utility function [14]. Discrete choice model parameters are estimated by a maximum likelihood estimation algorithm [15]. Different authors reviewed various discrete choice models and their applications $[14,16,17]$.

Empirical results demonstrate inadequacy of multinomial logit choice model for itinerary share prediction $[14,18]$. These results indicate that the itineraries which are closer to each other by departure time have great substitution or competition among them in comparison with others [19].

The modeling process of airline passengers' choice of itineraries is initially represented using MNL model [14]. According to this model, the main question is whether changing choice model from multinomial to nested logit during all conditions is reasonable? Does the sole concentration on the correlation among the products and results of statistical tests on the market data, which may lead to the instantaneous change of the primary choice model, necessarily increase revenue of the firm? In this paper, effort has been done to propose a new architecture for filling this gap.

Various factors such as modeler intuition and statistical tests lead to choosing the most appropriate choice model. During the routine process of choicebased revenue management, statistical tests are used for selecting suitable choice model and interaction of choice and optimization models are not considered in this process. According to the effects of the selected choice model in the complexity of optimization model, this paper proposes a system to balance expected revenue improvement obtained by incorporating more realistic choice model with the growth of complexity of algorithm.

Rule-based reasoning module is proposed in the new architecture for analyzing this interaction and selecting the most appropriate choice model. The main component of this module is a mechanism with the ability of prediction. The artificial neural network is developed for this purpose, which can predict the expected revenue improvement obtained by importing more realistic choice model in the optimization model.

Artificial Neural Networks (ANN) were inspired by the brain modeling studies. One of the main classes of applications to which artificial neural networks have been applied includes function approximation which learns the functional relationship between input and the desired output vectors. The outstanding advantage of this mechanism is its needlessness for advanced assumptions about the relationship between different factors and the ability to learn this relationship from the data itself [20].

Most of the research on using neural network in the revenue management models concentrate on forecasting the demand [21-30]. Volling et al. [31] used neural network for approximating the opportunity cost of resources. There are other works which concentrate on prediction of the customers' behavior by neural networks [32-34]. There is vast literature about using neural network in financial areas [27,3540]. Artificial neural network is also applied for travel time prediction [41-43]. Application of neural network in traffic flow prediction area is presented in another research [44,45]. We are planning to use neural network for predicting revenue improvement percent. This network should learn the impacts of effective factors, such as remaining capacity and time, on the obtained revenue, which change during booking horizon. According to this mechanism, during booking horizon, at the updating periods, choosing choice model process should be refreshed.

The remainder of this paper is organized as follows. In the next section, architecture of the proposed system and its components will be explained. This section describes the new process flow and its operations. In Section 3, the details of incorporating 
neural network in the proposed system are described. In Section 4, the results of implementing the new system in two networks and comparison with results of the obtained revenue in the former models are presented. The last section summarizes key findings of the paper.

\section{Model structure}

In this part, the main elements of the proposed conceptual model are described.

\subsection{Traditional revenue management process}

The general architecture of revenue management process flow includes different stages, such as forecasting and optimizing.

During the process of revenue management, suitable choice model is selected, forecasting is done, and optimization is run for deciding about the optimal decision variables. Although the structure of optimization problem is affected by the incorporated discrete choice model, the mentioned process ignores this interaction. This paper proposes the new system in order to make balance between increasing the complexity of the optimization problem and the expected revenue improvement obtained by incorporating more realistic and complex choice model in the optimization module.

\subsection{Components of the proposed system}

Figure 1 shows the architecture of the proposed intelligent system.

The main components of the proposed system are:
Data-management subsystem. The relevant data is available in the data-management subsystem. This subsystem can be connected with a repository, which is data warehouse, to corporate relevant decisionmaking data [46]. The required data warehouse in our model includes products data, such as available products, their prices and other properties of them, and market historical data. The market historical data stores behaviors and reactions of the customers to the available products and can be used for managing customer related processes.

Model management subsystem. This package includes different models that provide analytical capabilities of the system. This subsystem includes statistical models, optimization models, discrete choice models, and other relevant quantitative models.

User-interface subsystem. In our proposed system, the user should determine the threshold value. This value could be changed during different periods. The user communicates with and commands the system through user interface subsystem.

Knowledge-acquisition subsystem. This subsystem provides intelligence for the proposed system. The intelligence in our subsystem is provided by a neural network and a rule bank. In order to train the neural network, knowledge of the organization should be incorporated in the system. The rule bank is applied

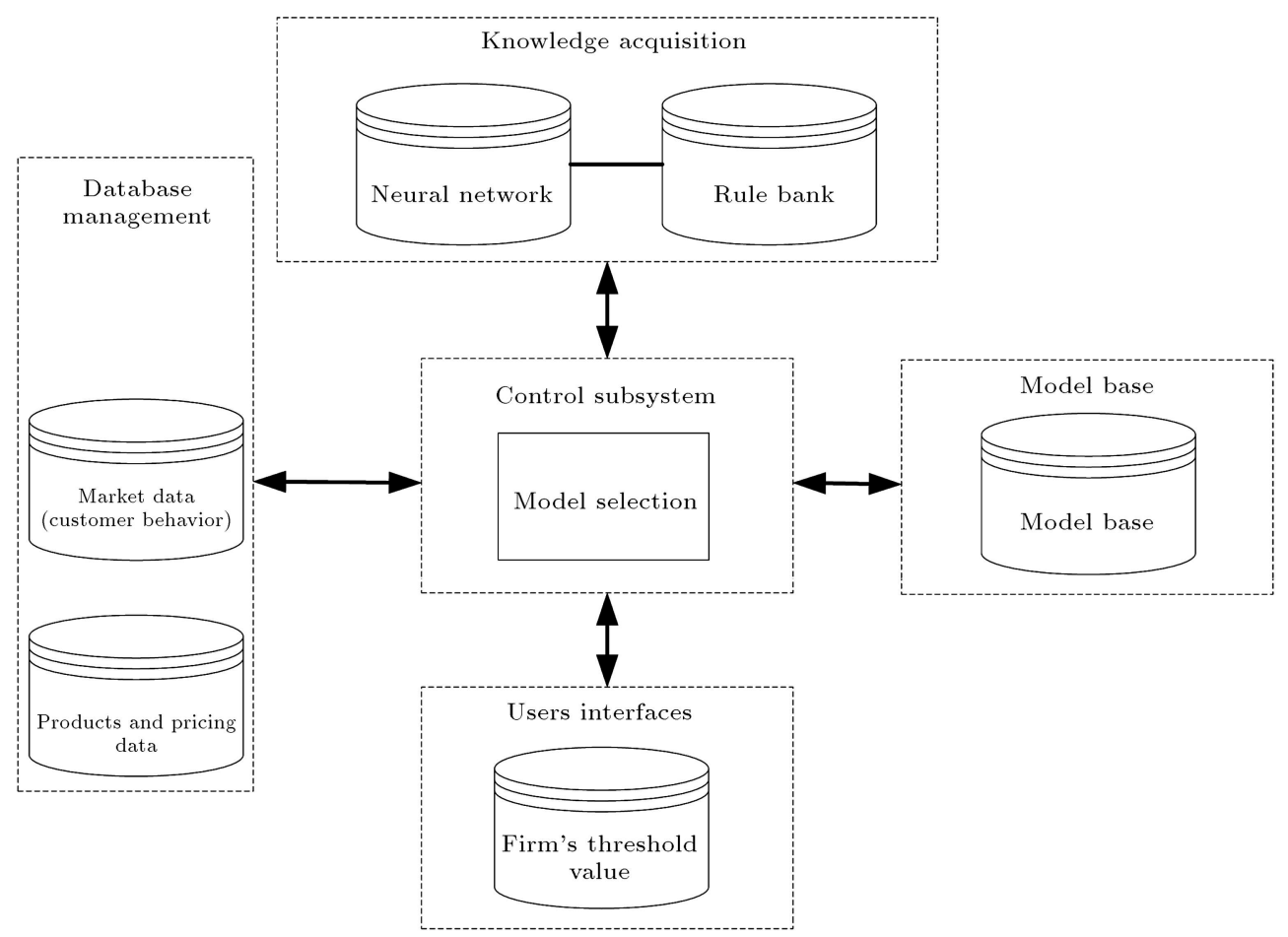

Figure 1. Architecture of the proposed intelligent system. 


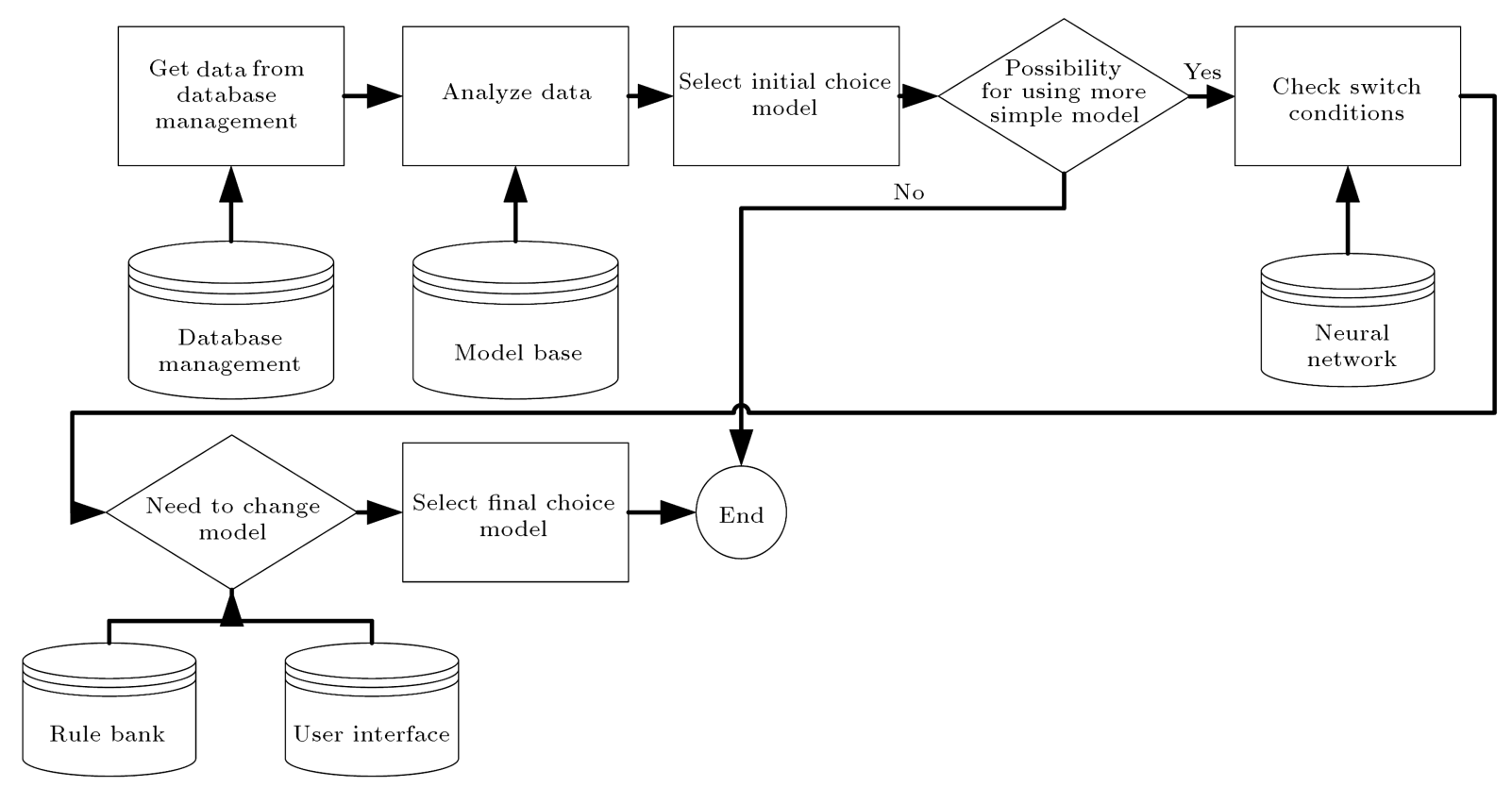

Figure 2. Process flow of the proposed system.

for deciding about the best choice model according to the user's threshold value.

Finally, control subsystem facilitates make communications between different components of this system. The details of the neural network will be explained in the next section. Figure 2 explains the process flow of this system.

At the beginning, the required initial data, such as information about products and customers, is extracted from data-management subsystem. Then, the process of selecting the most appropriate discrete choice model is run. It is assumed that multinomial logit is the basic choice model and the system will check whether it is necessary to switch from base model to a more complicated choice model. Statistical tests of the correlation will affect the decision. The result of this decision will not be submitted to the optimization module, immediately. Selecting the more complicated choice models will activate the knowledge acquisition subsystem. In this system, neural network predicts the expected improvement obtained by incorporating these choice models. This value is compared with the user's threshold value. The user's threshold value indicates the importance of the obtained results. In the condition of superiority of the expected improvement over the threshold value, the system will be convinced to incorporate more complex choice model in the optimization module.

Cycling this process during booking horizon would result in applying different choice models in the optimization module. Different factors such as the remaining capacity, remaining time periods, and the importance of decisions influence this process.

\subsection{Time complexity}

Solution approach for the optimization model is based on the proposed method for solving choice-based network revenue management by Bront et al. [12]. According to this method, the column generation algorithm is used for solving choice-based deterministic linear programming problem. A greedy heuristic algorithm is proposed for solving the subproblem of the column generation. Etebari et al. [7] described details of substituting multinomial by nested logit model in the choice-based deterministic linear programming. Bront et al. [12] demonstrate that the worst complexity of this problem by considering MNL in the heuristic algorithm is $O\left(n^{2} L\right)$, in which $n$ shows the number of products and $L$ indicates the number of segments in the model. By substituting the nested logit model by the multinomial logit, time complexity of the new heuristic will be $O\left(n^{2} N\right)$, in which $N$ is the number of nests. Therefore, time complexity of the new problem will be at least twice the previous one.

\section{Neural network model}

We propose neural network for predicting the revenue improvement obtained by importing more realistic choice model in the optimization model. The key motivation for doing so is the ability of neural networks to learn the functional form of the true model from the data without any prior assumption about them. The remaining capacity of each resource at the updating times constitutes input data for the model.

A neural network is the collection of nodes, the way they are connected, and the coefficients within the network that determine the intensity of the input 
signals, which is known as weights. Transfer function is an algorithmic process which transforms input to output. We should train the neural networks for adjusting their weights based on a learning function. In a supervised learning mode, the actual output of a neural network is compared to the desired output.

For the purpose of predicting the improvement percent, the Multi-Layer Perceptron (MLP) is considered. The mentioned network consists of three layers including an input layer, a hidden layer, and an output layer. The remaining capacity of each resource, $\left(x_{1}, x_{2}, \ldots, x_{m}\right)$ at the updating times is the input data of the model. The hidden nodes of the layer define the relationship between inputs and outputs. It is assumed that the number of hidden layer nodes is equal to the number of network's legs. The output is the expected improvement percent obtained by feeding more realistic choice model into the optimization model. We build a set of $N$ neural networks, each modeling the capacityrevenue improvement relationship for updating periods. We should define training sets for each neural network. Simulation is used for creating these sets according to two distinct scenarios. These scenarios are defined in a way that in both of them, it is supposed that there is correlation between the error terms of products utility. The first scenario assumes that the firm neglects the correlation and applies the multinomial logit model for specifying offer sets of the products; the next scenario assumes that the firm is using a more realistic and complex choice model which in this research is nested logit. This simulation is done for different values of capacities assumed for each leg, and the results specify the improvement percent.

Logsigmoid activation function is used in the hidden layer and pureline function is used in the output layer. For normalizing input variables, their values are transformed to numbers between 0 and 1 . Doing so, the transformed values, denoted by $x_{i}^{\prime}$, are given by the following equation:

$$
x_{i}^{\prime}=\frac{x_{i}-x_{i}^{\min }}{x_{i}^{\max }-x_{i}^{\min }}=\frac{x_{i}}{C_{i}},
$$

where $x_{i}^{\text {min }}$ is the minimum value of capacity of resources, equal to 0 , and $x_{i}^{\max }$ is the maximum value which equals to the initial capacity level of resources $\left(C_{i}\right)$.

In this network, the well-known backpropagation method is used for training the neural network. During this algorithm, the errors propagate backwards from the output nodes to the inner nodes. This algorithm calculates the gradient of the error of the network and these values are used in a simple stochastic gradient descent algorithm to find weights that minimize the errors. The errors are calculated by squaring the

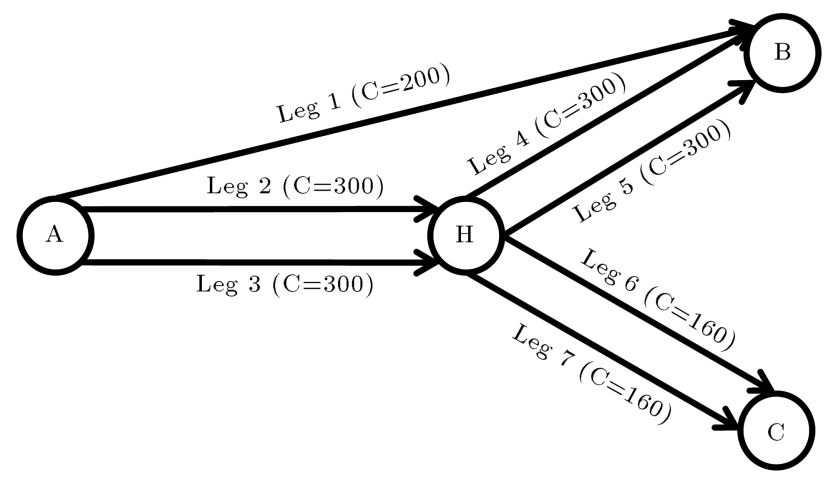

Figure 3. A small network airline.

difference between the actual and the desired outputs of the network. Levenberg-Marquardt backpropagation is used as a learning function for updating weights and biases.

\section{Experimental result}

The results of incorporating the proposed system in the two networks are described in this part.

\subsection{An airline network}

Figure 3 depicts the airline network. In this network, there are 7 legs with capacities $C=$ $(200,300,300,300,300,160,160)$. According to the itineraries and fare class combinations, 22 different products are defined in this network. It is assumed that the firm updates available products for two times during an offering horizon and it decides about the most suitable choice model. The objective of this system is deciding about offering products by keeping complexity of the system at a reasonable level. Tables 1 and 2 represent the defined products in this network.

Segmentation is done according to the price and time sensitivities and origin and destination of customers. Table 2 represents the segmentation.

The probability of customer's arrival, the nests, their consideration sets, and the observed utility for the products are represented, respectively. The firm should specify the threshold value for the system, which is supposed to be one percent in this problem. 300 different in put vectors are used for constituting training sets for each neural network and 100 iterations are done for each input vector. It is assumed that there are three different strategies which the firm should select among them. The first strategy is related to application of the simpler base choice model without considering the correlation in the nests. The next strategy is incorporating more realistic choice model in all periods. The last option is running the proposed system for deciding about the most suitable choice model.

Columns of Table 3 indicate the obtained revenue through the strategy of preferring the base multinomial choice model during all periods, switching to the more 
Table 1. Product definition for a small network problem.

\begin{tabular}{|c|c|c|c|c|c|c|c|}
\hline Products & Legs & Class & Fare & Products & Legs & Class & Fare \\
\hline 1 & 1 & $\mathrm{H}$ & 1000 & 12 & 1 & $\mathrm{~L}$ & 500 \\
\hline 2 & 2 & $\mathrm{H}$ & 400 & 13 & 2 & $\mathrm{~L}$ & 200 \\
\hline 3 & 3 & $\mathrm{H}$ & 400 & 14 & 3 & $\mathrm{~L}$ & 200 \\
\hline 4 & 4 & $\mathrm{H}$ & 300 & 15 & 4 & $\mathrm{~L}$ & 150 \\
\hline 5 & 5 & $\mathrm{H}$ & 300 & 16 & 5 & $\mathrm{~L}$ & 150 \\
\hline 6 & 6 & $\mathrm{H}$ & 500 & 17 & 6 & $\mathrm{~L}$ & 250 \\
\hline 7 & 7 & $\mathrm{H}$ & 500 & 18 & 7 & $\mathrm{~L}$ & 250 \\
\hline 8 & $\{2,4\}$ & $\mathrm{H}$ & 600 & 19 & $\{2,4\}$ & $\mathrm{L}$ & 300 \\
\hline 9 & $\{3,5\}$ & $\mathrm{H}$ & 600 & 20 & $\{3,5\}$ & $\mathrm{L}$ & 300 \\
\hline 10 & $\{2,6\}$ & $\mathrm{H}$ & 700 & 21 & $\{2,6\}$ & $\mathrm{L}$ & 350 \\
\hline 11 & $\{3,7\}$ & $\mathrm{H}$ & 700 & 22 & $\{3,7\}$ & $\mathrm{L}$ & 350 \\
\hline
\end{tabular}

Table 2. Customer segmentation in a small network problem.

\begin{tabular}{|c|c|c|c|c|c|c|c|c|c|c|c|}
\hline Segment & O-D & Nest & $\begin{array}{c}\text { Con. } \\
\text { set }\end{array}$ & Observed utility & $\lambda$ & Segment & O-D & Nest & $\begin{array}{c}\text { Con. } \\
\text { set }\end{array}$ & $\begin{array}{c}\text { Observed } \\
\text { utility }\end{array}$ & $\lambda$ \\
\hline \multirow[t]{2}{*}{1} & \multirow{2}{*}{ A-B } & 1 & $\{1,8,9\}$ & $(2.3000,2.0800,2.0800)$ & \multirow{2}{*}{0.08} & \multirow{2}{*}{6} & \multirow{2}{*}{ H-B } & 1 & $\{4,5\}$ & $(0.6900,0.6900)$ & \multirow[t]{2}{*}{0.15} \\
\hline & & 2 & $\{12,19,20\}$ & $(1.7900,1.3900,1.3900)$ & & & & 2 & $\{15,16\}$ & $(2.3000,2.0800)$ & \\
\hline \multirow{2}{*}{2} & \multirow{2}{*}{ A-B } & 1 & $\{1,8,9\}$ & $(0.0001,0.6900,0.6900)$ & \multirow{2}{*}{0.2} & \multirow{2}{*}{7} & \multirow{2}{*}{$\mathrm{H}-\mathrm{C}$} & 1 & $\{6,7\}$ & $(2.3000,2.0800)$ & \multirow{2}{*}{0.02} \\
\hline & & 2 & $\{12,19,20\}$ & $(2.0800,2.3000,2.3000)$ & & & & 2 & $\{17,18\}$ & $(1.6100,1.6100)$ & \\
\hline \multirow{2}{*}{3} & \multirow{2}{*}{ A-H } & 1 & $\{2,3\}$ & $(2.3000,2.3000)$ & \multirow{2}{*}{0.05} & \multirow{2}{*}{8} & \multirow{2}{*}{$\mathrm{H}-\mathrm{C}$} & 1 & $\{6,7\}$ & $(0.6900,0.6900)$ & \multirow{2}{*}{0.05} \\
\hline & & 2 & $\{13,14\}$ & $(1.6100,1.6100)$ & & & & 2 & $\{17,18\}$ & $(2.3000,2.0800)$ & \\
\hline \multirow{2}{*}{4} & \multirow{2}{*}{$\mathrm{A}-\mathrm{H}$} & 1 & $\{2,3\}$ & $(0.6900,0.6900)$ & \multirow{2}{*}{0.2} & \multirow{2}{*}{9} & \multirow{2}{*}{ A-C } & 1 & $\{10,11\}$ & $(2.3000,2.0800)$ & \multirow{2}{*}{0.02} \\
\hline & & 2 & $\{13,14\}$ & $(2.3000,2.3000)$ & & & & 2 & $\{21,22\}$ & $(1.6100,1.6100)$ & \\
\hline \multirow{2}{*}{5} & \multirow{2}{*}{ H-B } & 1 & $\{4,5\}$ & $(2.3000,2.3000)$ & \multirow{2}{*}{0.1} & \multirow{2}{*}{10} & \multirow{2}{*}{$\mathrm{A}-\mathrm{C}$} & 1 & $\{10,11\}$ & $(0.6900,0.6900)$ & \multirow{2}{*}{0.04} \\
\hline & & 2 & $\{15,16\}$ & $(1.6100,1.6100)$ & & & & 2 & $\{21,22\}$ & $(2.3000,2.3000)$ & \\
\hline
\end{tabular}

Table 3. The obtained revenue for the firm according to different strategies for choosing choice model.

\begin{tabular}{ccccc}
\hline \multirow{2}{*}{ Initial capacity } & \multicolumn{4}{c}{ Correlation=0.4 } \\
\cline { 2 - 5 } & $\begin{array}{c}\text { Multinomial } \\
\text { logit }\end{array}$ & $\begin{array}{c}\text { Nested } \\
\text { logit }\end{array}$ & DSS & $\begin{array}{c}\text { No. of } \\
\text { switch }\end{array}$ \\
\hline$[120,180,180,180,180,96,96]$ & 417153 & 422493 & 422280 & 2 \\
{$[160,240,240,240,240,128,128]$} & 517511 & 518906 & 517391 & 0 \\
{$[200,300,300,300,300,160,160]$} & 550523 & 550595 & 550349 & 0 \\
\hline
\end{tabular}

complicated nested logit model all times, and finally applying the proposed decision support system for choosing the most appropriate choice model, respectively. The mentioned numbers in the last column show the number of times in which the firm decides to incorporate nested logit model in the optimization module. Notice that in this problem, there are two times in which the firm updates optimization problem and should decide about choice model.

95\% confidence interval improvement percent by incorporating different choice models during the mentioned strategies are represented in Table 4. The NLM is related to the strategy to which the firm prefers to apply more realistic choice model during all periods. The MNL is always concerned with using the base choice model. DSS illustrates the strategy by which the proposed system decides about the most appropriate choice model.

Table 3 demonstrates that capacity restriction amplifies the importance of choosing the most realistic 
Table 4. $95 \%$ confidence interval for revenue improvement percent while applying different strategies for choosing choice model.

\begin{tabular}{ccc}
\hline \multirow{2}{*}{ Initial capacity } & \multicolumn{2}{c}{ Correlation=0.4 } \\
\cline { 2 - 3 } & NLM-MNL & NLM-DSS \\
\hline$[120,180,180,180,180,96,96]$ & $(0.72,1.91)$ & $(-0.39,0.63)$ \\
{$[160,240,240,240,240,128,128]$} & $(-0.30,0.89)$ & $(-0.47,0.65)$ \\
{$[200,300,300,300,300,160,160]$} & $(-0.59,0.67)$ & $(-0.51,0.79)$ \\
\hline
\end{tabular}

Table 5. The obtained revenue for the firm according to different strategies for choosing choice model.

\begin{tabular}{ccccc}
\hline & \multicolumn{4}{c}{ Correlation=0.6 } \\
\cline { 2 - 5 } Initial capacity & $\begin{array}{c}\text { Multinomial } \\
\text { logit }\end{array}$ & $\begin{array}{c}\text { Nested } \\
\text { logit }\end{array}$ & DSS & $\begin{array}{c}\text { No. of } \\
\text { switch }\end{array}$ \\
\hline$[120,180,180,180,180,96,96]$ & 409932 & 420047 & 419896 & 2 \\
{$[160,240,240,240,240,128,128]$} & 504693 & 510283 & 510032 & 2 \\
{$[200,300,300,300,300,160,160]$} & 538365 & 540045 & 539747 & 1 \\
\hline
\end{tabular}

Table 6. $95 \%$ confidence interval for revenue improvement percent while applying different strategies for choosing choice model.

\begin{tabular}{ccc}
\hline \multirow{2}{*}{ Initial capacity } & \multicolumn{2}{c}{ Correlation=0.6 } \\
\cline { 2 - 3 } & NLM-MNL & NLM-DSS \\
\hline$[120,180,180,180,180,96,96]$ & $(1.83,3.19)$ & $(-0.59,0.69)$ \\
{$[160,240,240,240,240,128,128]$} & $(0.44,1.87)$ & $(-0.35,0.79)$ \\
{$[200,300,300,300,300,160,160]$} & $(-0.31,1.01)$ & $(-0.53,0.69)$ \\
\hline
\end{tabular}

Table 7. The obtained revenue for the firm according to different strategies for choosing choice model.

\begin{tabular}{ccccc}
\hline \multirow{2}{*}{ Initial capacity } & \multicolumn{3}{c}{ Correlation=0.8 } \\
\cline { 2 - 5 } & Multinomial logit & Nested logit & DSS & No. of switch \\
\hline$[120,180,180,180,180,96,96]$ & 377209 & 415616 & 415954 & 2 \\
{$[160,240,240,240,240,128,128]$} & 469113 & 484492 & 484009 & 2 \\
{$[200,300,300,300,300,160,160]$} & 503605 & 523203 & 523189 & 2 \\
\hline
\end{tabular}

Table 8. $95 \%$ confidence interval for revenue improvement percent while applying different strategies for choosing choice model.

\begin{tabular}{ccc}
\hline & \multicolumn{2}{c}{ Correlation=0.8 } \\
\cline { 2 - 3 } Initial capacity & NLM-MNL & NLM-DSS \\
\hline$[120,180,180,180,180,96,96]$ & $(9.42,11.07)$ & $(-0.27,0.46)$ \\
{$[160,240,240,240,240,128,128]$} & $(2.64,3.99)$ & $(-0.38,0.74)$ \\
{$[200,300,300,300,300,160,160]$} & $(3.20,4.67)$ & $(-0.28,0.39)$ \\
\hline
\end{tabular}

choice model. In the Table 3 , in which the correlation is less than those in other tables, in the second and third rows, the reasoning module prefers to use base choice model. The results indicate that switching to the nested logit model, according to statistical tests results without considering structure of the optimization model, will increase the complexity of the problem, which does not necessarily improve revenue of the firm. Table 4 indicates that although conducting statistical tests demonstrates significant correlation, decision of the proposed system to use the base simple choice model preserves revenue of the firm, while decreasing complexity of the system.

Moreover, concentrating on Tables 5 and 6 , it is remarkable that increasing correlation of the nest results in increase in the total number of switches to the more complicated choice model.

Subsequent tables refer to the results of increasing correlation measure in the nests.

Tables 7 and 8 show that high scarce capacity and high correlation measure lead to applying the more realistic choice model during all updating periods. 


\section{Conclusion}

In this paper, an intelligent decision support system is proposed for selecting and incorporating the most appropriate choice model in the optimization module. According to the effects of applied choice model on the structure of optimization module, the routine revenue management process ignores this interaction. Following this process ends in the results that indicate this ignorance would increase time complexity of optimization problem without significant increase in the obtained revenue. This system focuses on balancing additional expected revenue obtained by incorporating more realistic choice model in the optimization model with time complexity of the obtained optimization problem.

The main part of this system is knowledge acquisition subsystem. The main component of this subsystem is a neural network. After selecting the discrete choice model based on the revealed data and statistical tests of the current market, neural network is used for analyzing the interaction between choice and optimization models. This leads to prediction of the revenue improvement obtained by feeding more realistic choice model into the optimization module. These outcomes are compared with the firm's threshold value that states importance of the final decision. Thus, the final decision is made about the most suitable discrete choice model.

Computation results indicate that in spite of fitting market data with the nested logit model, it is not necessary to switch from the simple multinomial choice model to the more complex nested logit during all conditions. Furthermore, the results reveal that reasoning module leads to decrease in complexity of the problem by applying simpler choice models, while preserving revenue of the firm. These results show that when there are scarce capacity and high correlation, incorporating a more realistic choice model is of paramount importance. This process should be updated at repeated intervals and the selected choice model could change during booking horizon.

\section{References}

1. Cross, R.G., Revenue Management: Hard-Core Tactics for Market Domination, Broadway Books, New York (1997).

2. Talluri, K.T. and Van Ryzin, G., The Theory and Practice of Revenue Management, New York: Kluwer Academic Publishers (2004).

3. Schon, C. "Optimal dynamic price selection under attraction choice models", European Journal of $\mathrm{Op}$ erational Research, 205(3), pp. 650-660 (2010).

4. Belobaba, P. and Hopperstad, C. "Boeing/MIT simulation study: PODS results update", in AGIFORS
Reservation and Yield Management Study Group Symposium Proceedings, London (1999).

5. Zhang, D. and Cooper, W.L. "Revenue management for parallel flights with customer-choice behavior", Operations Research, 53(3), pp. 415-431 (2005).

6. Van Ryzin, G. and Vulcano, G. "Computing virtual nesting controls for network revenue management under customer choice behavior", Manufacturing \& Service Operations Management, 10(3), pp. 448-467 (2008).

7. Etebari, F., Aghaei, A. and Jalalimanesh, A. "Network revenue management under specific choice models", Scientia Iranica, 20(6), pp. 2161-2176 (2013).

8. Chen, L. and Homem-de-Mello, T. "Mathematical programming models for revenue management under customer choice", European Journal of Operational Research, 203(2), pp. 294-305 (2010).

9. Talluri, K. and Van Ryzin, G. "Revenue management under a general discrete choice model of consumer behavior", Management Science, 50, pp. 15-33 (2004).

10. Gallego, G., et al. "Managing flexible products on a network", Department of Industrial Engineering and Operations Research, Columbia University (2004).

11. Liu, Q. and Van Ryzin, G. "On the choice-based linear programming model for network revenue management", Journal of Manufacturing \& Service Operations Management, 10, pp. 288-311 (2008).

12. Bront, J.J.M., Méndez-Díaz, I. and Vulcano, G. "A column generation algorithm for choice-based network revenue management", Operations Research, 57(3), pp. 769-784 (2009).

13. Meissner, J. and Strauss, A. "Improved bid prices for choice-based network revenue management", European Journal of Operational Research, 217, pp. 417-427 (2012).

14. Garrow, L.A., Discrete Choice Modelling and Air Travel Demand, Georgia Institute of Technology, USA: Ashgate publishing company (2010).

15. Vulcano, G., van Ryzin, G. and Chaar, W. "Choicebased revenue management: An empirical study of estimation and optimization", Manufacturing \& Service Operations Management, 12, pp. 371-392 (2010).

16. Ben-Akiva, M.E. and Lerman, S.R., Discrete Choice Analysis: Theory and Application to Travel Demand, 9, The MIT Press (1985).

17. Train, K.E., Discrete Choice Methods with Simulation, New York: Cambridge University Press (2009).

18. Coldren, G.M. and Koppelman, F.S. "Modeling the competition among air-travel itinerary shares: GEV model development", Transportation Research Part A, 39, pp. 345-365 (2005).

19. Coldren, G.M. and Koppelman, F.S. "Modeling the proximate covariance property of air travel itineraries along the time-of-day dimension", Transportation Research Record, 1915, pp. 112-123 (2005). 
20. Engelbrecht Anderson, P., Computational Intelligence, Wiley (2007).

21. Shakya, S., Kern, M., Owusu, G. and Chin, C.M. "Neural network demand models and evolutionary optimisers for dynamic pricing", Knowledge-Based Systems, 29, pp. 44-53 (2012).

22. Tsai, T.-H., Lee, C.-K. and Wei, C.-K. "Neural network based temporal feature models for shortterm railway passenger demand forecasting", Expert Systems with Applications, 36, pp. 3728-3736 (2009).

23. Padhi, S.S. and Aggarwal, V. "Competitive revenue management for fixing quota and price of hotel commodities under uncertainty", International Journal of Hospitality Management, 30, pp. 725-734 (2011).

24. Borisov, P.A., Vinogradov, G.P. and Semenov, N.A. "Integration of neural network algorithms, nonlinear dynamics models, and fuzzy logic methods in prediction problems", Journal of Computer and Systems Sciences International, 47, pp. 78-84 (2008).

25. Stella, F., Vigano, V., Bogni, D. and Benzoni, M. "An integrated forecasting and regularization framework for light rail transit systems", Journal of Intelligent Transportation Systems, 10, pp. 59-73 (2007).

26. Seyedabrishami, S. and Shafahi, Y. "Expert knowledge-guided travel demand estimation: Neuro-fuzzy approach", Journal of Intelligent Transportation Systems: Technology, Planning, and Operations, 15, pp. 13-27 (2011).

27. Nekoukar, V. and Beheshti, M. "A local linear radial basis function neural network for financial time-series forecasting", Applied Intelligence, 33, pp. 352-356 (2010).

28. Mantin, B. and Koo, B. "Weekend effect in airfare pricing", Journal of Air Transport Management, 16, pp. $48-50$ (2010).

29. Van Hinsbergen, C.P.I.J., Hegyi, A., van Lint, J.W.C. and van Zuylen, H.J. "Bayesian neural networks for the prediction of stochastic travel times in urban networks", IET Intelligent Transportation Systems, $\mathbf{5}$, pp. 259-265 (2011).

30. Yaghini, M., Khoshraftar, M.M. and Seyedabadi, M. "Railway passenger train delay prediction via neural network model", Journal of Advanced Transportation, 47, pp. 193-203 (2012).

31. Volling, T., Akyol, D.E., Wittek, K. and Spengler, T.S. "A two-stage bid-price control for make-to-order revenue management", Computers and Operations Research, 39, pp. 1021-1032 (2012).

32. Hruschka, H., Ferres, W. and Probst, M. "An empirical comparison of the validity of a neural net based multinomial logit choice model to alternative model specifications", European Journal of Operations Research, 159, pp. 166-180 (2004).

33. West, P.M., Brocket, P.L. and Golden, L.L. "A comparative analysis of neural networks and statistical methods for predicting consumer choice", Marketing Science, 16, pp. 370-391 (1997).
34. Yang, H., Kitamura, R., Jovanis, P.P., Vaughn, K.M. and Abdel-Aty, M.A. "Exploration of route choice behavior with advanced traveler information using neural network concepts", Transportation, 20, pp. 199223 (1993).

35. Falavigna, G. "Financial ratings with scarce information: A neural network approach", Expert Systems with Applications, 39, pp. 1784-1792 (2012).

36. Rodríguez-González, A., García-Crespo, Á., ColomoPalacios, R., Iglesias, F.G. and Gómez-Berbís, J.M. "CAST: Using neural networks to improve trading systems based on technical analysis by means of the RSI financial indicator", Expert Systems with Applications, 38, pp. 11489-11500 (2011).

37. Guresen, E., Kayakutlu, G. and Daim, T.U. "Using artificial neural network models in stock market index prediction", Expert Systems with Applications, 38, pp. 10389-10297 (2011).

38. Liao, Z. and Wang, J. "Forecasting model of global stock index by stochastic time effective neural network", Expert Systems with Applications, 37, pp. 834841 (2010).

39. Abu-Mostafa, Y.S. and Atiya, A.F. "Introduction to financial forecasting", Applied Intelligence, 6, pp. 205213 (1996).

40. Kim, K. and An, H. "Simultaneous optimization of artificial neural networks for financial forecasting", Applied Intelligence, 36, pp. 887-898 (2012).

41. Mazlumi, E., Rose, G., Currie, G. and Sarvi, M. "An integrated framework to predict bus travel time and its variability using traffic flow data", Journal of Intelligent Transportation Systems, 15, pp. 75-90 (2011).

42. Bin, Y., Zhongzhen, Y. and Baozhen, Y. "Bus arrival time prediction using support vector machines", Journal of Intelligent Transportation Systems, 10, pp. 151158 (2007).

43. Innamaa, S. "Short-term prediction of travel time using neural networks on an interurban highway", Transportation, 32, pp. 649-669 (2005).

44. Abdulhai, B., Porwal, H. and Recker, W. "Short-term traffic flow prediction using neuro-genetic algorithms", Journal of Intelligent Transportation Systems, 7, pp. 3-41 (2002).

45. Dia, H. and Panwai, S. "Neural agent (Neugent) models of driver behavior for supporting ITS simulations", International Journal of Intelligent transportation Systems Research, 9, pp. 23-36 (2011).

46. Turban, E., Aronson, J.E. and Liang, T.P., Decision Support Systems and Intelligent Systems, Prentice Hall of India (2007).

\section{Biographies}

Farhad Etebari received his BS degree in Industrial Engineering from Sharif University of Technology and 
his $\mathrm{MS}$ and $\mathrm{PhD}$ degrees in Industrial Engineering from K. N. Toosi University of Technology. His research interests include optimization models, logistic systems, choice-based revenue management, transportation planning, and AI algorithms.

Amir Abbas Najafi was born in Tehran, Iran 1974. $\mathrm{He}$ received his BS degree in Industrial Engineering from Isfahan University of Technology in 1996, and his $\mathrm{MS}$ and $\mathrm{PhD}$ degrees in Industrial Engineering from Sharif University of Technology in 1998 and 2005, respectively. His research interests include investment management, project management, EPQ model with scrapped items and limited production capacity, economic and financial analysis, inventory control, and applied operations research. 